\title{
Planungstheorie und Planungswissenschaft im Praxistest: Arbeitsalltag und Perspektiven von Regionalplanern in Deutschland
}

\author{
Meike Hellmich $^{1}$ Christian Wilhelm Lamker ${ }^{2}$ Linda Lange $^{3}$
}

Eingegangen: 31. März 2016 / Angenommen: 15. November 2016 / Online publiziert: 23. November 2016

(c) Springer-Verlag Berlin Heidelberg 2016

Zusammenfassung Räumliche Herausforderungen überschreiten zunehmend kommunale Grenzen und Regionalplaner stehen in komplexen Zusammenhängen zwischen lokaler Selbstverwaltung und großräumigen Veränderungen, in denen sie mit formellen und informellen Instrumenten gleichermaßen agieren können. Doch wie überhaupt geplant wird und wie der Arbeitsalltag eines Regionalplaners in Deutschland aussieht, ist wissenschaftlich nur wenig erforscht. Auch die Einstellung von Praktikern gegenüber der Planungswissenschaft und planungstheoretischen Ansätzen ist vielfach unklar und wenig empirisch belegt. Diesen Fragestellungen widmet sich der vorliegende Beitrag, der auf einer bundesweiten Online-Umfrage von Regionalplanern in Deutschland beruht. Ebenso vielfältig wie die Planung als Disziplin sind die ihr zugrunde liegenden Theorien und

\footnotetext{
Anmerkung Die empirische Arbeit, die diesem Artikel zugrunde liegt, wurde organisatorisch durch die Akademie für Raumforschung und Landesplanung (ARL) und die Technische Universität Dortmund, Fakultät Raumplanung, unterstützt.

Meike Hellmich

meike.hellmich@thuenen.de

$\triangle$ Dr. Christian Wilhelm Lamker christian.lamker@tu-dortmund.de

Linda Lange

lange@umwelt.uni-hannover.de

1 Institut für Ländliche Räume, Johann Heinrich von Thünen-Institut Braunschweig, Bundesallee 50, 38116 Braunschweig, Deutschland

2 Fachgebiet Stadt- und Regionalplanung, Technische Universität Dortmund, August-Schmidt-Straße 10, 44227 Dortmund, Deutschland

3 Institut für Umweltplanung, Leibniz Universität Hannover, Herrenhäuser Straße 2, 30419 Hannover, Deutschland
}

Praktiken, mit denen geplant und umgesetzt wird. Eine bessere Wissensgrundlage über den aktuellen Planungsalltag und die Einschätzungen von Regionalplanern ist erforderlich, um deren Möglichkeiten einschätzen und sie wissenschaftlich zielgerichtet unterstützen zu können. In diesem Beitrag werden Fragen zum Arbeitsalltag deutscher Regionalplaner, ihrem aktuellen Planungshandeln, dem Transfer von Erkenntnissen und Expertisen zwischen Wissenschaft und Praxis, der Entstehung und dem Nutzen von Theorien sowie der Sichtweise der Planungspraktiker auf Planungstheorien behandelt. Die Ergebnisse zeigen eine vielfältige Landschaft praktischer Tätigkeiten und eine Dualität zwischen großem Interesse an wissenschaftlichen Erkenntnissen und Planungstheorie, aber auch Kommunikations- und Verständnisprobleme auf.

Schlüsselwörter Planungswissenschaft . Planungstheorie · Planungspraxis · Theorie-PraxisTransfer $\cdot$ Regionalplanung $\cdot$ Befragung

\section{Planning Theory and Planning Science in Practice: Everyday Work and Prospects for Regional Planners in Germany}

Abstract Spatial planning challenges increasingly cross
local boundaries. Statutory planning, which is organized
along all different levels of government, is very complex
and with its mix of informal and formal instruments diffi-
cult to put into fixed categories. It acts between local self-
autonomy and large-scale spatial changes. Available empir-
ical evidence about how and whereby planning is currently
done, is often unclear or weak. This article is grounded on
a survey of practitioners within regional planning adminis-
trations in Germany to investigate the development and use 
of theories and the perspectives of practitioners on these theories. Theories and their understanding prove to be as diverse as practice itself. The challenge to translate scientific evidence into working progresses of practitioners, the understanding of theories and the self-perception of planning practitioners has also been addressed. One main aim of this article is to show perspectives on the daily work in practice, the transfer of expertise between research and practice, the emergence and use of theories as well as the perception of planning theories by practitioners. Results reveal a highly diverse landscape of planning practices and a duality between a vested interest in research evidence and planning theories, but also deficiencies in communication and mutual understanding.

Keywords Planning research · Planning theory · Planning practice $\cdot$ Theory-practice transfer $\cdot$ Regional planning . Survey

\section{Hintergrund und Motivation}

Die Frage, wie in der Praxis der räumlichen Planung tatsächlich gehandelt wird und in welchem Verhältnis Planungswissenschaft, Planungstheorie und Planungspraxis stehen, ist so alt wie die Disziplin der Planung selbst (vgl. z. B. Faludi 1973; Yiftachel 1989; Alexander 1997; MacDonald/Sanyal/Silver et al. 2014; Davoudi 2015). Öffentliche Planung beinhaltet formelle Abwägungsprozesse für räumliche Belange, Festsetzungen auf unterschiedlichen Verwaltungsebenen, raumwirksame Beschlüsse mit unterschiedlichen Bindungswirkungen ebenso wie informelle Planungs- und Beteiligungsverfahren. Räumliche Planungsprozesse sind komplex und in ihren Handlungsspielräumen immer situations- und kontextabhängig. Für die Zukunft werden in Deutschland unter anderem Energiewende, Klimawandel, Öffentlichkeitsbeteiligung und Bürgeremanzipation, Digitalisierung, Kooperation, gelebte Demokratie und die Legitimation von Planung selbst als Trends benannt, die in den Arbeitsalltag von Planern hineinwirken (vgl. ARL 2014). Die raum- und planungsbezogene Wissenschaft hat dabei in den letzten Jahrzehnten verstärkt den Anspruch entwickelt, möglichst praxisbezogene Themen aufzugreifen und dem Vorwurf der ,realitätsfernen“
Untersuchungen durch angewandte Forschungsmethoden und transdisziplinäre Forschungsansätze entgegenzuwirken (Zierhofer/Burger 2007; Renn 2008; Blotevogel/Wiegand 2015).

Das Bestreben, die Verbindungen zwischen Wissenschaft, Theorie und Praxis näher zu beleuchten, wird an einer steigenden Anzahl von wissenschaftlichen Artikeln und Projekten deutlich. Diese widmen sich entweder den Unzulänglichkeiten von Theorien an sich (z. B. Alexander 2010; Lord 2014; MacDonald/Sanyal/Silver et al. 2014; Alexander 2016) oder der Verbindung zwischen Planungswissenschaft und Planungspraxis (z. B. Davoudi 2015; Hurley/Lamker/Taylor et al. 2016; Taylor/Hurley 2016). Aber auch wissenschaftliche Diskussionsrunden sind zu diesem Thema etabliert, wie etwa der im Jahr 2013 von der Akademie für Raumforschung und Landesplanung (ARL) eingesetzte Arbeitskreis ,Mind the Gap - Kooperationen und Selbstverständnisse in der räumlichen Planung“. In diesem Arbeitskreis wurde sich der beschriebenen Fragestellung auf verschiedene Arten mit mehreren Schwerpunkten genähert, von denen der vorliegende Beitrag die Perspektive aus der Planungspraxis einbringt.

Die inhärente Interdisziplinarität von Planung bedingt vielfältige theoretische Annäherungen an gesellschaftliche und politische Systeme (Peters 2004: 5). Zur Einordnung gebraucht wird ein gutes Bild davon, wie Planungspraxis tatsächlich handelt. Was fehlt, ist eine breite und aktuelle empirische Basis als Grundlage für weitere Diskussionen und Schlussfolgerungen für das Verhältnis und Zusammenwirken von Theorie, Wissenschaft und Praxis.

Ausgangspunkt dieses Beitrags und der zugrunde liegenden Befragung ist die Annahme, dass die Untersuchung und Diskussion entlang der Felder Theorie, Wissenschaft und Praxis strukturiert werden kann (vgl. Abbildung 1). Diese Felder existieren nebeneinander und können im Folgenden aus der Perspektive von Praktikern tiefer ergründet werden. Dabei ist Planungstheorie grundsätzlich zu verstehen als theoretischer Hintergrund der räumlichen Planung, der auf abstrahierten gedanklichen Konstrukten aufbaut. Diese gedanklichen Konstrukte sind auch aus der Planungspraxis denkbar, wenn davon ausgegangen wird, dass alle Planer eine eigene Vorstellung ihres Handelns und ein eigenes Fundament dafür haben (vgl. z. B. Whittemore 2015). Der Begriff Planungstheorie ist in dieser Dreiteilung al-
Abbildung 1 Zusammenhänge zwischen Planungstheorien, Planungswissenschaft und Planungspraxis (Quelle: Lamker 2016: 209, verändert)

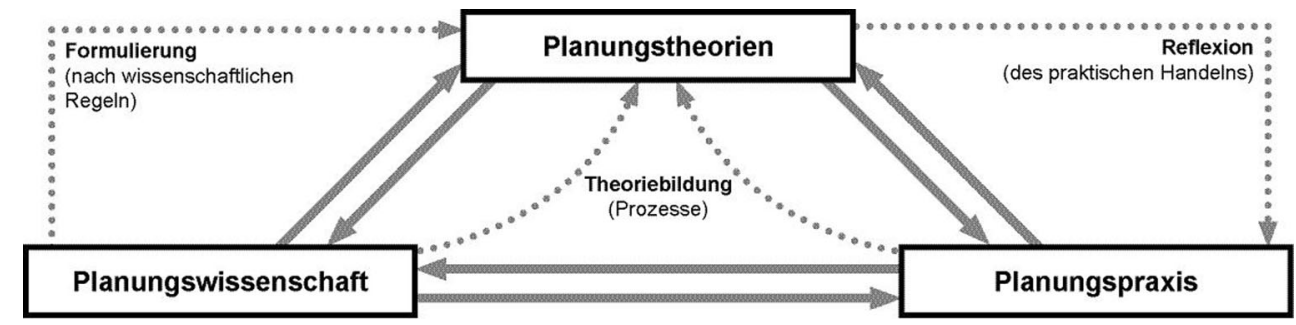


so nicht der Wissenschaft vorbehalten, sondern kann auch aus der Praxisperspektive gedacht und empirisch ergründet werden. Formuliert werden Planungstheorien nach wissenschaftlichen Regeln in der Planungswissenschaft und stehen dann auch wiederum als Reflexionsgrundlage für praktisches Handeln zur Verfügung (vgl. Needham 2004: $238 \mathrm{f}$.; Beauregard 2013: 474). Unter Planungspraxis wird hingegen zunächst das Handeln von Personen gefasst, die in einer öffentlichen Planungsstelle tätig sind und mit Belangen der räumlichen Entwicklung befasst sind. Die Wissenschaft kann zwischen Theorie und Praxis als eine Übersetzungsebene fungieren. Dies bedeutet, dass sich die Wissenschaft auf der einen Seite der Planungstheorien bedient und auf der anderen Seite ihr Wissen in die Praxis einfließen lässt und umgekehrt. Planungstheorien stehen in einer Verbindung sowohl zur Wissenschaft als auch zur Praxis und in einer gemeinsamen Verbindung von Prozessen der Theoriebildung (vgl. auch Whittemore 2015: 82). Eine integrative Herangehensweise ist in der Planungswissenschaft zwingend notwendig, da sie nicht auf eine Erprobung von Lösungsund Erklärungsansätzen wie etwa in der naturwissenschaftlichen Grundlagenforschung zurückgreifen kann. Auf der einen Seite steht die Wissenschaft somit als Übersetzer für die Erschließung von Planungstheorien durch Praktiker in ihrer Alltagsarbeit. Auf der anderen Seite prüft die Wissenschaft mit ihrer angewandten Herangehensweise eigene Annahmen und Hypothesen anhand von Beobachtungen der Planungspraxis.

Der Fokus dieses Beitrags liegt auf der Darstellung der empirischen Arbeit und somit auch auf einer Beleuchtung der Praxis (Kapitel 2). Eine vertiefte und sich von der Befragung lösende Reflexion über das Verhältnis von Planungstheorie und Planungspraxis ist damit weiteren Arbeiten vorbehalten, zu denen dieser Beitrag Denkanstöße liefern soll. Der Beitrag orientiert sich in seiner Struktur an der Abfolge des entwickelten Fragebogens und greift die dort behandelten Schwerpunkte auf. Dabei geht es darum, Antworten auf die folgenden Fragen zu ergründen:

- Wie sieht der Alltag eines Planers in der regionalen Planung in Deutschland aus?

- Wie werden wissenschaftliche Erkenntnisse im Planungsalltag genutzt?

- Welche Auffassung von Planungstheorien vertreten Praktiker?

Die Befragung liefert einen Ausschnitt aus der Perspektive der Planungspraxis in drei Bereichen (Kapitel 3). Sie gibt erstens einen Einblick, wie sich die Praxis aktuell überhaupt darstellt und in ihrem Handeln beschreiben lässt. Zweitens beleuchtet sie, wie die Praxis auf die Wissenschaft und die Theorie blickt. Der dritte Fokus liegt auf der Zusammenarbeit zwischen Planungspraxis und Planungswissenschaft. Aus der Analyse heraus sind beschränkte Rückschlüsse zu- dem auf Prozesse der Theoriebildung sowie auf die Nutzbarkeit von Planungstheorien für praktisches Handeln möglich (Kapitel 4). Praxis meint bei dieser Untersuchung die Praxis der öffentlichen Regionalplanung in Deutschland, die beispielhaft für eine Planungspraxis steht, die deutschlandweit flächendeckend betrieben wird. Die Motivation ist, herauszufinden, ob bzw. wo Unterschiede zwischen der alltäglichen Planungspraxis und den theoretischen Verständnissen und Vorstellungen existieren bzw. wie sich die aktuelle Planungspraxis gestaltet und welchen Herausforderungen sich die Praxis zukünftig nach Einschätzung der Befragten stellen muss. Wenn Planungstheorien (mindestens) das Ziel haben oder dazu dienen sollen, abstrakt und reflexiv eine Planungspraxis zu erklären, ergänzt dieser Einblick die verfügbaren empirischen Grundlagen und Bausteine und regt zudem weitergehende Diskussionen an (Kapitel 5).

\section{Methodische Konzeption und Durchführung der Studie}

\subsection{Aufbau und Inhalte}

Um eine breite empirische Basis aus der Planungspraxis zu erhalten und die vom Begriff her bereits handlungsorientierte Planung näher zu untersuchen, bietet sich die Ebene von Handlungen von Planungsakteuren an, nach denen mithilfe eines Fragebogens gefragt werden kann. Persönliche Einstellungen, kognitive Muster sowie individuelle Gedankengänge, die zwar für die Auseinandersetzung mit der Thematik interessant sein könnten, lassen sich jedoch nur über narrative Einzelinterviews generieren (vgl. Küsters 2009). Sie wurden hier nicht berücksichtigt. Die Befragung unter dem Titel "Die Praxis der Regionalplanung in Deutschland“ sollte einen breiteren Einblick über Einzelfälle hinaus bieten. Sie wurde Anfang 2015 online mit sechs Frageblöcken durchgeführt: Angaben zur Institution (1), Planerische Prozesse (2), Planerisches Handeln (3), Planerische Grundlagen und Austausch zwischen Planungspraxis und Wissenschaft (4), Planungstheorie und Planungspraxis (5), Persönliche Angaben (6).

Die Angaben zur Institution (1) im ersten Fragenblock dienen der allgemeinen Einordnung der Antworten und sind vor allem aufgrund der unterschiedlichen Verfasstheit der Regionalplanung und einer sehr unterschiedlichen Größe von Planungsregionen sowie der unterschiedlichen Personalausstattung von Trägern der Regionalplanung in Deutschland erforderlich. Im zweiten Teil der planerischen Prozesse (2) geht es um den zeitlichen Anteil gängiger planerischer Tätigkeiten im Arbeitsalltag der befragten Personen. Es wird beispielsweise gefragt, welchen zeitlichen Anteil die Aufstellung von Regionalplänen, das Engagement in Netzwerken oder das Beantragen und Ver- 
walten von Fördermitteln einnimmt. Bei den zeitlichen Angaben handelt es sich um eigene Einschätzungen wie „gering“, „hoch“ usw. Die zeitlichen Einstufungen sind nicht in Stunden oder Anteile der Arbeitszeit definiert, sondern werden durch den Befragten nach eigenem Ermessen interpretiert. Im dritten Abschnitt (3) zum planerischen Handeln wird erneut nach der Bedeutung von Tätigkeiten heute und der erwarteten Entwicklung in etwa 15 Jahren gefragt, dem üblichen Zeithorizont für Raumordnungspläne in Deutschland. Aus den zugrunde gelegten groben Rollenbildern der Planungstheorie ergeben sich 22 Verben, die als Antwortoptionen in diesem Fragenblock zur Bewertung gestellt werden. Dies sind beispielsweise Analysieren, Beurteilen, Erforschen, Koordinieren, Moderieren oder Verwalten. Abschließend wird in diesem dritten Abschnitt in Form einer offenen Frage ein Rückblick auf die zurückliegenden 15 Jahre und die gegebenenfalls stattgefundenen Veränderungen in der Planungspraxis eingeholt. Der vierte Frageblock (4) widmet sich der Unterstützung der Planungspraxis durch die Wissenschaft, verstanden als Brücke zwischen Theorie und Praxis. Dabei geht es vor allem um die aktive Wahrnehmung und Nutzung von wissenschaftlichen Erkenntnissen sowie die Beteiligung der Planungspraxis an Forschungsprojekten. Der fünfte Teil (5) dient dazu, konkreter nach der Wahrnehmung und Bedeutung von Planungstheorien für die Planungspraxis zu fragen. Dieser Teil hat eine direkte Anknüpfung an die Planungstheorie und fordert die Teilnehmer zum Abschluss zu einem reflektierenden Denken heraus. Dazu werden gezielte Aussagen sowie ergänzende Definitionen von (Planungs-)Theorie zur Bewertung vorgeschlagen. Diese Statements wurden durch eine Arbeitsgruppe innerhalb des Arbeitskreises ,Mind the Gap - Kooperationen und Selbstverständnisse in der räumlichen Planung" der ARL als Diskussionsgrundlage entwickelt. Sie basieren auf einer Auswertung bestehender nationaler und internationaler Grundlagenliteratur zur Planungstheorie. Die Befragten geben an, ob sie der jeweiligen These voll, eher oder nicht zustimmen. Zum Abschluss werden im sechsten Teil (6) persönliche Angaben abgefragt.

Die Frageblöcke (1) Angaben zur Institution und (6) Persönliche Angaben werden als Vergleichsvariablen hinzugezogen, um mögliche generationenbedingte Unterschiede oder Abhängigkeiten und Unterschiede in den Antworten beispielsweise aufgrund der Stellung im Beruf oder dem fachlichen Hintergrund zu erkennen und entsprechend einordnen zu können. Die Ergebnisse aus diesen Frageblöcken fließen somit übergreifend in die Ergebnisdarstellungen der Frageblöcke (2) bis (5) mit ein und werden nicht separat erläutert.

\subsection{Durchführung und Rücklauf}

Für die Durchführung der Onlinebefragung wurde der Befragungsserver der Fakultät Raumplanung an der Technischen Universität Dortmund genutzt, auf dem eine aktuelle LimeSurvey-Installation $^{1}$ vorhanden ist. Um die Neutralität und Unabhängigkeit der Befragung zu verdeutlichen, wurde eine für alle Teilnehmer neutrale Adresse für die Website (www.planungsbefragung.de) eingerichtet und verwendet.

Mögliche Kontaktdaten, Ansprechpartner und Träger der Regionalplanung wurden auf bundesweiter Ebene überwiegend über die ARL und via Internetrecherche ausfindig gemacht und aktualisiert. Vor der Einladung der Probanden und der finalen Aktivierung der Befragung wurde ein umfangreicher Pretest der Online-Fragebogenversion durchgeführt, um den Fragebogen auf Verständlichkeit, inhaltliche Stringenz und technische Durchführbarkeit zu prüfen. Hierzu wurde der Fragebogen mit Experten aus Wissenschaft und Praxis ${ }^{2}$ diskutiert und entsprechend angepasst. Außerdem wurde der Fragebogen im Vorlauf der Befragung auf der 4. Deutschen Regionalplanungstagung der ARL im September 2014 in Würzburg einem breiten Publikum aus Wissenschaft und Praxis vorgestellt, diskutiert und beworben.

Dem Fragebogen wurde ein Einleitungsschreiben vorgeschaltet, welches neben dem inhaltlichen Fokus zudem Hinweise zur technischen Bearbeitung enthielt. Die Datenerhebung erfolgte vollständig anonym. Nach vier Wochen Befragungszeitraum wurde eine postalische Erinnerung versendet, um den Rücklauf zu steigern. Insgesamt wurden 156 Personen per E-Mail direkt angeschrieben und zur Teilnahme an der Befragung aufgefordert. Um einen möglichst großen Kreis an potenziellen Probanden generieren zu können, wurde zusätzlich darum gebeten, die Befragung auch an Kollegen aus dem eigenen Hause, die in der Regionalplanung arbeiten, weiterzuleiten. Insgesamt konnten 59 vollständig ausgefüllte Fragebögen verzeichnet werden. Die Grundgesamtheit aller Regionalplaner in Deutschland ist nicht bekannt, sodass keine genaue Rücklaufquote angegeben werden kann. Ausgehend von der Zahl verschickter Einladungen ergibt sich ein Rücklauf von etwa 38 Prozent.

\subsection{Auswertung quantitativer und qualitativer Daten}

Die Auswertung erfolgte zunächst quantitativ mithilfe deskriptiver Statistik nach dem Export der Daten aus LimeSurvey in Microsoft Excel sowie SPSS (Statistical Package

\footnotetext{
${ }^{1}$ LimeSurvey: frei verfügbarer Webservice, um Online-Umfragen auch ohne Programmierkenntnisse zu entwickeln und zu veröffentlichen.

${ }^{2}$ Der Arbeitskreis, in den die Befragung eingebettet war, setzt sich zusammen aus Planungswissenschaftlern und Planungspraktikern.
} 
Tabelle 1 Heutiger Aufwand für planerische Aufgaben (absolute Häufigkeiten, n=59)

\begin{tabular}{|c|c|c|c|c|c|}
\hline Formelle Tätigkeiten & hoch & eher hoch & eher gering & gering & k. A. \\
\hline Aufstellung von Regionalplänen & 35 & 16 & 5 & 3 & 0 \\
\hline $\begin{array}{l}\text { Organisation/Durchführung von formellen Beteiligungsverfah- } \\
\text { ren }\end{array}$ & 20 & 18 & 17 & 4 & 0 \\
\hline Beteiligung an formellen Stellungnahmen & 15 & 29 & 14 & 1 & 0 \\
\hline Sitzungsvorbereitung für politische Gremien & 15 & 25 & 12 & 6 & 1 \\
\hline $\begin{array}{l}\text { Organisation/Durchführung behördeninterner Abstimmungs- } \\
\text { verfahren }\end{array}$ & 6 & 19 & 31 & 3 & 0 \\
\hline Durchführung von Raumordnungsverfahren & 3 & 9 & 14 & 31 & 2 \\
\hline Koordination/Bearbeitung von Zielabweichungsverfahren & 3 & 7 & 16 & 31 & 2 \\
\hline Monitoring/Umsetzungskontrolle von Regionalplänen & 2 & 14 & 25 & 18 & 0 \\
\hline Sonstiges & 1 & 2 & 5 & 3 & 48 \\
\hline Informelle Tätigkeiten & hoch & eher hoch & eher gering & gering & k.A. \\
\hline Austausch mit anderen Behörden der eigenen Planungsregion & 16 & 33 & 10 & 0 & 0 \\
\hline Aufbau/Stärkung von regionalen Netzwerken & 7 & 22 & 21 & 9 & 0 \\
\hline Nutzung/Gestaltung informeller Instrumente & 5 & 26 & 20 & 8 & 0 \\
\hline Beantragung oder Verwaltung von Fördermitteln & 2 & 11 & 20 & 26 & 0 \\
\hline Austausch mit anderen Regionalplanungsstellen & 1 & 24 & 29 & 5 & 0 \\
\hline Engagement in landes- oder bundesweiten Netzwerken & 0 & 9 & 27 & 23 & 0 \\
\hline Engagement in internationalen Netzwerken & 0 & 2 & 15 & 42 & 0 \\
\hline
\end{tabular}

for the Social Sciences). Im ersten Schritt wurden die geschlossenen Fragen deskriptiv ausgewertet und reine Häufigkeitsanalysen durchgeführt. In einem zweiten Auswertungsschritt wurden Korrelationen zu den abgefragten persönlichen Angaben und Rahmendaten hergestellt, um beispielsweise zu prüfen, welchen Einfluss das Bundesland, das Alter oder die Position auf das Antwortverhalten haben. Die qualitativen Daten aus den offenen Antwortfeldern wurden in MaxQDA ${ }^{3}$ aufbereitet und verarbeitet. Die Antworten wurden dazu in einem Codesystem klassifiziert (kodiert), gruppiert und entsprechend ausgewertet.

\section{Perspektiven aus dem aktuellen Regionalplanungsalltag}

Die Intention dieses Beitrags liegt darin, das Verhältnis zwischen Praxis, Wissenschaft und Theorie genauer zu beleuchten und aus der Sicht regionaler Planungspraktiker darzustellen. Dafür ist es notwendig, den Alltag der Praktiker, den Austausch mit der Wissenschaft und die Sichtweise auf Planungstheorien empirisch zu erfassen.

\subsection{Planerische Prozesse heute und in Zukunft - zwischen formellen und informellen Tätigkeiten}

In dem Fragenblock zu planerischen Prozessen wird nach dem zeitlichen Anteil gängiger planerischer Tätigkeiten im Arbeitsalltag der befragten Personen und der erwarte-

\footnotetext{
${ }^{3}$ MaxQDA: Visualisierungsprogramm qualitativer Analysen.
}

ten Entwicklung in etwa 15 Jahren gefragt, dem üblichen Zeithorizont für Raumordnungspläne in Deutschland. Dadurch wird ein erster Beitrag geliefert, um die „Praxis der räumlichen Planung“ besser erklären und darstellen zu können. Als Antwortmöglichkeit gibt es sowohl formelle (z. B. Durchführung von Raumordnungsverfahren) als auch informelle (z. B. Beteiligung an informellen Beteiligungsverfahren) Prozesse bzw. Schwerpunkte, welche nach aktuellen Erkenntnissen in das Aufgabengebiet eines Regionalplaners passen.

In den Antworten wird deutlich, dass formelle Planungstätigkeiten den größten zeitlichen Anteil im derzeitigen Arbeitsalltag einnehmen. Etwa zwei Drittel der Regionalplaner geben an, einen hohen zeitlichen Anteil des planerischen Handelns mit der Aufstellung von Regionalplänen $\mathrm{zu}$ verbringen (vgl. Tabelle 1). Jeweils etwa ein Drittel der Befragten gibt zudem an, einen hohen zeitlichen Anteil des Arbeitsalltages mit der Durchführung von formellen Beteiligungsverfahren, formellen Stellungnahmen sowie der Sitzungsvorbereitung für politische Gremien zu verbringen. Zudem nimmt der Austausch mit anderen Behörden der eigenen Planungsregion ebenfalls einen sehr hohen zeitlichen Anteil ein. $83 \%$ geben hier ein „hoch“ oder ,eher hoch“ an. Auffallend bei den Antwortangaben ist außerdem, dass fast alle der vorgegebenen Antwortmöglichkeiten - wenn auch in unterschiedlicher Nennungsanzahl - einen hohen zeitlichen Anteil (sehr hoch oder eher hoch) im Planungsalltag einnehmen. Als weniger bedeutend werden die Punkte „Fördermittel beantragen“, „Austausch mit anderen Regionalplanungsstellen“ sowie „Engagement in internationalen Netzwerken“ eingestuft. 
Tabelle 2 Anteil planerisches Handeln heute (absolute Häufigkeiten, $\mathrm{n}=59$ )

\begin{tabular}{llllll}
\hline hoch & $\begin{array}{l}\text { eher } \\
\text { hoch }\end{array}$ & $\begin{array}{l}\text { eher } \\
\text { gering }\end{array}$ & gering & k. A. \\
Argumentieren & 24 & 29 & 6 & 0 & 0 \\
Beurteilen & 21 & 35 & 1 & 0 & 2 \\
Koordinieren & 21 & 30 & 7 & 0 & 1 \\
Diskutieren & 20 & 30 & 9 & 0 & 0 \\
Schreiben & 18 & 29 & 10 & 2 & 0 \\
Planen & 16 & 31 & 8 & 4 & 0 \\
Analysieren & 15 & 31 & 12 & 0 & 1 \\
Überzeugen & 15 & 31 & 11 & 0 & 2 \\
Festlegen & 14 & 29 & 13 & 2 & 1 \\
Managen & 13 & 20 & 18 & 4 & 4 \\
Moderieren & 13 & 25 & 14 & 6 & 1 \\
Unterstützen & 13 & 30 & 12 & 3 & 1 \\
Beobachten & 10 & 30 & 14 & 4 & 1 \\
Hinterfragen & 9 & 35 & 14 & 0 & 1 \\
Verwalten & 7 & 17 & 26 & 6 & 3 \\
Ordnen & 6 & 23 & 24 & 4 & 2 \\
Entwickeln & 5 & 34 & 15 & 3 & 2 \\
Zeichnen & 5 & 13 & 12 & 29 & 0 \\
(auch digital) & & & & & \\
Sichern & 4 & 25 & 23 & 4 & 3 \\
Kritisieren & 3 & 18 & 30 & 5 & 3 \\
Erforschen & 1 & 1 & 26 & 29 & 2 \\
Navigieren & 1 & 16 & 23 & 10 & 9 \\
\hline
\end{tabular}

Werden die persönlichen Angaben der Befragten (z. B. Alter oder Position innerhalb der Planungsstelle) der Befragten einbezogen, lässt sich ein Zusammenhang zwischen der Positions eines Planers mit den zeitlichen Anteilen seiner Tätigkeiten feststellen: Hohe Hierarchiestufen sind stärker mit den Tätigkeiten informeller planerischer Prozesse, dem Engagement in Netzwerken und der Fördermittelakquise verbunden. Formelle Stellungnahmen sowie behördeninterne Abstimmungsverfahren durchziehen gleichmäBig alle Hierarchiestufen.

In Bezug auf die erwarteten Veränderungen im Alltag wird angegeben, dass sich die aktuellen planerischen Tätigkeiten zukünftig nicht substanziell ändern werden. Bereits heute häufige planerische Tätigkeiten werden etwas an Bedeutsamkeit gewinnen. Das Monitoring und die Umsetzungskontrolle formeller Planungsinstrumente sowie die Bedeutung informeller Planungsverfahren werden nach Einschätzung der befragten Planungspraktiker zukünftig in ihrem zeitlichen Anteil zunehmend wichtiger. Etwa die Hälfte der Befragten gibt an, dass die Nutzung und Gestaltung informeller Instrumente eine höhere Bedeutung haben wird. Auch die informellen Beteiligungsverfahren werden nach deren Einschätzung weiter zunehmen. Eher an Bedeutung verlieren werden sowohl das Engagement in internationalen als auch in bundesweiten Netzwerken.

\subsection{Argumentieren, Beurteilen und Koordinieren - der Planer heute}

Unter dem Bereich „planerisches Handeln“ werden gängige planerische Tätigkeiten und deren Bedeutung im heutigen Planungsalltag in Form von Verben abgefragt, um mögliche Rollenbilder des Planers aufzudecken. Außerdem wird die erwartete Entwicklung dieser Handlungsweisen für einen Zeithorizont von 15 Jahren abgefragt, da Veränderungen im planerischen Handeln sich mitunter bereits heute andeuten. Es wird deutlich, dass das Beurteilen, Argumentieren sowie koordinierende Handlungen aktuell den größten zeitlichen Anteil der planerischen Verhaltensweisen einnehmen (vgl. Tabelle 2). Navigieren und Erforschen stellen hingegen nur einen geringen Anteil dar.

Werden diese Antworten in Beziehung zu den allgemeinen Angaben der Befragten gesetzt (Alter und Ausbildung), fällt auf, dass ein höherer Berufsabschluss einen substanziell höheren Anteil an Managementaufgaben und unterstützenden Tätigkeiten im aktuellen Planungsalltag bedeutet. Ebenso wird durch die vorliegenden Ergebnisse deutlich, dass das Argumentieren und Moderieren für diese Gruppe der Befragten einen hohen Anteil am planerischen Handeln ausmacht.

Im Bereich der zu erwartenden Veränderungen in einem Zeithorizont von 15 Jahren sind keine grundlegenden Unterschiede festzustellen. Die größte Zunahme wird vor allem bei kommunikativen Verhaltensweisen (Argumentieren, Moderieren und Überzeugen) erwartet. Ebenso wie im Kapitel 3.1 „Planerische Prozesse“ werden planerische Tätigkeiten, die schon heute einen großen zeitlichen Anteil einnehmen, zukünftig auch weiter an Bedeutsamkeit gewinnen.

\subsection{Von der Wissenschaft in die Praxis und zurück}

Dieser Fragenblock widmet sich der Unterstützung der Planungspraxis durch die Wissenschaft, verstanden als eine Brücke zwischen Theorie und Praxis. Dabei geht es vor allem um die aktive Wahrnehmung von wissenschaftlichen Erkenntnissen sowie die eigene Beteiligung an Forschungsprojekten. Regelmäßig werden demnach Fachzeitschriften gelesen und Fachkonferenzen sowie fachbezogene Websites besucht (vgl. Tabelle 3). Die häufigsten überhaupt nicht genutzten Informationsquellen sind die Fortbildungen bei Berufsverbänden oder Kammern, persönliche Kontakte in die Wissenschaft sowie die Beteiligung an Forschungsprojekten. Obwohl $71 \%$ der befragten Personen angeben, in den letzten Jahren die aktive Beteiligung in einem wissenschaftlichen Forschungsprojekt als Informationsquelle genutzt zu haben, werden Ergebnisdokumentationen von Forschungsprojekten sowie Fachbücher deutlich seltener gelesen. 
Tabelle 3 Genutzte Informationsquellen für wissenschaftliche Erkenntnisse (absolute Häufigkeiten, n=59)

\begin{tabular}{|c|c|c|c|c|}
\hline & regelmäßig & gelegentlich & gar nicht & k. A. \\
\hline Fachbezogene Websites & 35 & 22 & 1 & 1 \\
\hline Fachzeitschriften & 29 & 30 & 0 & 0 \\
\hline Fachkonferenzen & 29 & 29 & 1 & 0 \\
\hline Fortbildungen der Verwaltungen & 25 & 24 & 9 & 1 \\
\hline Integration/Austausch in fachbezogenen Netzwerken & 25 & 25 & 9 & 0 \\
\hline Einstellung von Praktikanten/studentischen Hilfskräften & 22 & 23 & 13 & 1 \\
\hline Zeitschriften von Berufsverbänden & 19 & 32 & 8 & 0 \\
\hline Website der ARL & 12 & 39 & 8 & 0 \\
\hline Persönliche Kontakte in die Wissenschaft & 12 & 29 & 18 & 0 \\
\hline Fachbücher & 11 & 37 & 11 & 0 \\
\hline Fortbildungen bei Berufsverbänden oder Kammern & 11 & 27 & 21 & 0 \\
\hline Ergebnisdokumentationen zu Forschungsprojekten & 11 & 36 & 11 & 1 \\
\hline Beteiligung in Forschungsprojekten & 8 & 34 & 17 & 0 \\
\hline Sonstiges & 0 & 0 & 1 & 58 \\
\hline
\end{tabular}

Einfacher Zugang ist eine Grundvoraussetzung (vgl. auch Hurley/Lamker/Taylor et al. 2016: $449 \mathrm{f}$.), damit wissenschaftliche Erkenntnisse in der Praxis überhaupt wahrgenommen und berücksichtigt werden. Im Überblick zeigt sich deutlich, dass leicht zugängliche Informationsquellen häufiger regelmäßig genutzt werden. Die Befragung hat hier keine weitere Untergliederung nach Art der Fachzeitschriften oder Konferenzen vorgenommen. Zeitund arbeitsintensive Quellen wie die aktive Beteiligung in Forschungsprojekten werden eher gelegentlich genutzt. Die offenen Antworten deuten darauf hin, dass hier sowohl Zeitgründe wie auch Probleme in der thematischen Passfähigkeit und der Einpassung wissenschaftlicher Empfehlungen in praktische Restriktionen (vor allem politische Unterstützung und öffentliche Diskussionen) wichtige Gründe sind. Der persönliche Austausch wird allgemein hoch geschätzt, wenn diese individuellen, thematischen und politischen Hürden überwunden werden. Lange textliche Ausarbeitungen in Büchern oder Ergebnisberichten werden insgesamt am seltensten als Informationsquellen genutzt.

Die allgemeine Unterstützung der eigenen Arbeiten durch die Wissenschaft schätzen die befragten Planungspraktiker allerdings als eher gering ein; dies bezieht sich sowohl auf den Bereich der Planungsprozesse und -methoden als auch der Planungsinhalte. $66 \%$ schätzen die wissenschaftliche Unterstützung für Planungsprozesse als gering oder eher gering ein, $59 \%$ für Planungsmethoden und immer noch $53 \%$ für Planungsinhalte. Die Angaben zum Unterstützungswunsch der Praxis durch die Wissenschaft sind ambivalent. Ein Teil der Befragten spricht sich deutlich für mehr Unterstützung aus; ein anderer Teil benötigt keine weitere Unterstützung. Hier kam mehrfach der Hinweis, dass einerseits gar keine Zeit im Arbeitsalltag bleibt, um sich mit wissenschaftlichen Erkenntnissen auseinanderzusetzen. Andererseits wurde darauf hingewiesen, dass wissenschaftliche Themen häufig nicht praxisrelevant sind und die Sprache der Wissenschaft unverständlich ist. Befragte weisen in diesem Zusammenhang explizit darauf hin, dass sie sich Hilfe für ,wirkliche Problemstellungen“ (ein Planer aus Niedersachsen) statt akademischer Diskussionen wünschen. Oder sie sehen wichtige Themen in der Praxis, die wissenschaftlich jedoch vernachlässigt werden. Genannt wurden unter anderem Regiopolen, die Schnittstelle von Regionalplanern zu Politikern sowie kritische Perspektiven, die zu einer Vereinfachung von Verfahren führen können (Planer aus Niedersachen und Mecklenburg-Vorpommern). Gewünscht wurde auch ein regelmäBiges länderübergreifendes Netzwerk zu Planungsthemen, in dem man sich wissenschaftliches Wissen einholen kann (ein Planer aus Thüringen).

\subsection{Planungstheorien - Zwischen Elfenbeinturm und praktischer Unterstützung?}

Bisher wurde der Begriff Planungstheorie als abstrakte Hülle verwendet. Planungstheorien gibt es in der Wissenschaft und der Praxis, sie unterscheiden sich von praktischem Handeln und es ist wichtig, Theorien nicht unabhängig von Wissenschaft oder Praxis zu denken (vgl. Alexander 2010: 103 f.; Lord 2014: 39). Wenn Gemeinsamkeiten und Unterschiede konkreter beschrieben und Verbindungen gestärkt werden sollen, erfordert das ein Verständnis davon, was bei Planungspraktikern unter Planungstheorie verstanden wird. Im Rahmen der Befragung wurden in einem ersten Schritt innerhalb des oben genannten Arbeitskreises aus der Aufarbeitung von Grundlagenliteratur und empirischen Arbeiten zum Verhältnis von Planungstheorie und Planungspraxis zehn Thesen über Planungstheorie entwickelt. Diesen haben die Befragten in einer vierstufigen Skala zugestimmt oder widersprochen. Die Beurteilung dieser Thesen wird 
Tabelle 4 Planungstheorie aus der Sicht der Planungspraktiker (absolute Häufigkeiten, n=59)

\begin{tabular}{|c|c|c|c|c|c|}
\hline & $\begin{array}{l}\text { stimme voll } \\
\text { zu }\end{array}$ & $\begin{array}{l}\text { stimme } \\
\text { eher zu }\end{array}$ & $\begin{array}{l}\text { stimme } \\
\text { eher nicht } \\
\text { zu }\end{array}$ & $\begin{array}{l}\text { stimme } \\
\text { nicht zu }\end{array}$ & k. A. \\
\hline $\begin{array}{l}\text { 6. Theoretisches Wissen ist nicht direkt in Handlungen über- } \\
\text { setzbar }\end{array}$ & 15 & 30 & 9 & 2 & 3 \\
\hline $\begin{array}{l}\text { 9. Praktische Realitäten widersprechen theoretischen Ansprü- } \\
\text { chen }\end{array}$ & 14 & 30 & 7 & 3 & 5 \\
\hline 7. Theorien können das Wissen der Planenden erweitern & 11 & 35 & 7 & 1 & 5 \\
\hline 10. Theorien sind zu abstrakt und schwer verständlich & 9 & 22 & 21 & 1 & 6 \\
\hline 3. In der Theoriebildung gibt es ,blinde Flecken` & 8 & 27 & 7 & 0 & 17 \\
\hline $\begin{array}{l}\text { 8. Allen Theorien liegen normative Ideale und Diskurse zu- } \\
\text { grunde }\end{array}$ & 7 & 18 & 11 & 0 & 23 \\
\hline $\begin{array}{l}\text { 4. Die Theoriebildung ist nicht hinreichend transparent und } \\
\text { selbstreflektiert }\end{array}$ & 4 & 22 & 13 & 2 & 18 \\
\hline $\begin{array}{l}\text { 2. Vorherrschende Planungstheorien bilden die Vielfalt von } \\
\text { Praktiken nicht ab }\end{array}$ & 3 & 38 & 6 & 2 & 10 \\
\hline 1. Planungstheoretiker entwerfen kritische Gegenbilder & 2 & 25 & 11 & 3 & 18 \\
\hline $\begin{array}{l}\text { 5. Planungstheorien beziehen sich zu stark auf } \\
\text { (Planungs-)Theorien }\end{array}$ & 1 & 22 & 11 & 2 & 23 \\
\hline
\end{tabular}

in Tabelle 4 aufgeschlüsselt. Die Nummerierung stellt die Reihenfolge der Abfrage dar.

Das Antwortverhalten ist sehr differenziert und folgt keinem Muster, das mit der Reihenfolge der Statements übereinstimmt. Die höchste Zustimmung erreichen kritische Aussagen. Praktiker sehen Schwierigkeiten in der Übersetzung von theoretischem Wissen in praktische Handlungen (6) und Widersprüche zwischen den theoretischen Ansprüchen und ihren praktischen Realitäten (9). Sie sehen aber auch das Potenzial von Theorien, ihr Wissen zu erweitern (7). Die Entstehung von Planungstheorien und die Beschäftigung damit sind für Praktiker schwierig. Die Aussagen 1, 4, 5 und 8 haben die höchste Zahl nicht abgegebener Einschätzungen (zwischen 31 und $39 \%$ ). Mehr als die Hälfte der Befragten ist der Meinung, dass die vorherrschenden Planungstheorien viele Praktiken nicht abbilden. Es scheint auch eine große Herausforderung zu sein, ,blinde Flecken' in der Theoriebildung zu erkennen und zu bearbeiten sowie die Vielfalt der Praktiken abzubilden und theoretische Erkenntnisse in die Praxis zurückzuspiegeln. Die geringste Zustimmung unter den Befragten gibt es dazu, dass Theorien zu abstrakt und schwer verständlich sind. Zwar gibt es auch hier noch eine mehrheitliche $\mathrm{Zu}$ stimmung (53\%), aber es scheint mehr Schwierigkeiten in den bearbeiteten Themen und dem Bezug zum eigenen Handeln zu geben als in der grundsätzlichen Verständlichkeit. Die Berührungsangst mit dem Begriff Planungstheorie ist geringer als vermutet, dennoch sind auf der Grundlage der Ergebnisse nur erste Ansätze für Rückschlüsse auf das jweilige eigene Verständnis von Planungstheorien möglich.

Um einer Antwort darauf, was Praktiker unter Planungstheorie verstehen, einen Schritt näher zu kommen, wurde allen Teilnehmern die Frage gestellt: „Welches Verständnis haben Sie von Planungstheorien?““. Insgesamt 30 Personen haben daraufhin ein eigenes Verständnis abgegeben. Für die Auswertung wurde versucht, die Antworten in die Schemata von Faludi (1973) und Allmendinger (2009) einzuordnen. In der Unterscheidung zwischen substanziellen und prozeduralen Planungstheorien nach Faludi (1973) konnten jeweils neun Antworten einer dieser Kategorien zugeordnet werden. Für Faludi sind beide Bereiche der Planungstheorie notwendig für eine effektive Planung, können aber analytisch getrennt werden in die eher rahmenbildende prozedurale Planungstheorie und die inhaltliche substanzielle Planungstheorie (Faludi 1973: $3 \mathrm{ff}$.). Eine abgegebene Antwort hat in einer längeren Definition beide Varianten abgedeckt, aber insgesamt elf Antworten konnten gar nicht zugeordnet werden. Viele beziehen sich in der Erläuterung ihres eigenen Verständnisses auf die Zusammenarbeit zwischen Wissenschaft und Praxis, auf Probleme bei der Anwendung von Theorien oder auf Restriktionen im Planungsalltag.

Allmendinger (2009: 11) unterscheidet Theorietypen nach normativen, präskriptiven und empirischen Theorien, Modellen, konzeptuellen Rahmen oder Perspektiven und die Theoriebildung. Normative Theorien geben Werte und Normen dazu wieder, wie etwas sein sollte. Präskriptive Theorien beinhalten Mittel zur Zielerreichung und haben dabei auch eine prognostische Komponente. Empirische Theorien sind in erster Linie deskriptive Erklärungen der Realität. In der Unterscheidung nach Allmendinger (2009) war die Mehrzahl der Verständnisse eindeutig normativpräskriptiv (12 Antworten) und nur in Einzelfällen deskriptiv oder explanativ. Von Planungstheorien wird in erster Linie eine Unterstützung für die (eigene) Planungspraxis erwartet. Auch nach diesem Schema von Allmendinger konnten 14 Antworten nicht zugeordnet werden. Dazu 
zählen Aussagen wie „Das Leben ist stärker“, die zwar eine klare Position beziehen, aber keine Rückschlüsse darauf zulassen, welches Theorieverständnis der Antwortende selbst hat. Ob sich die Befragten hierbei ein eigenes Modell, einen konzeptionellen Rahmen gebildet haben oder selbst in die Theoriebildung einsteigen, ist aus den kurzen Antworten nicht abzuleiten. Im Umkehrschluss heißt das, dass anerkannte Typisierungen von Planungstheorien einen Nutzen für die wissenschaftliche Beschäftigung mit Theorien und deren präzise Formulierung haben können, aber die Hürde für die Zusammenarbeit mit der Praxis durch ihre analytische Auftrennung eher höher setzen (vgl. auch die Kritik von Yiftachel 1989: 37 und Beauregard 2013: 476).

Ein Regionalplaner aus Baden-Württemberg stellt dazu Folgendes fest: „Planungstheorien bringen Ordnung in die Vielzahl der Gegenstände planerischen Handelns. Sie zeigen Beziehungen auf, die in der alltäglichen Arbeit manchmal übersehen werden und dann zu Überraschungen führen. Sie ersetzen Intuition durch Reflektion." Dieses beispielhafte Verständnis stellt einen weiteren Aspekt von Planungstheorien dar: auf Unerwartetes hinweisen sowie planerisches Handeln ordnen und reflektieren. Damit wird auch anerkannt, dass es sich bei Theorien um eine andere Sichtweise auf die Praxis handelt und dass sie komplementär zur Praxis sein können (vgl. van de Ven/Johnson 2006: 802 f.). Planungstheorien haben in der Praxis eine größere Basis als zunächst vermutet, um kreativ über imaginäre Zukünfte nachzudenken und auf bisher unbekannte Felder hinzuweisen (vgl. Vogelij 2015: 131). Sie müssen sich dann aber wiederum aktiver dem Problem von Verständlichkeit und Anknüpfungsfähigkeit an aktuelle vielfältige Planungspraktiken stellen (vgl. Fainstein/DeFilippis 2016: 14). Dieses vielschichtige Bild zeichnet sich auch in den Befragungsergebnissen ab: Viele Praktiker können durchaus mit dem Theoriebegriff arbeiten und sehen einen Wert in Planungstheorien, sehen aber Probleme in der Verknüpfung zu ihrer eigenen Tätigkeit. Die offenen Antworten machen auf der anderen Seite deutlich, dass Planungstheorie aus der Praxis (noch) schwieriger zu definieren ist und dass Praktiker nur eine unscharfe Trennlinie zwischen Wissenschaft und Theorie ziehen oder die Arbeit von Wissenschaftlern mit Theorie gleichsetzen. Planungstheorie darf somit durchaus über Luftschlösser nachdenken, aber dabei die Erdung in der Praxis nicht vergessen.

\section{Diskussion der gewonnenen Erkenntnisse}

Die Erkenntnisse aus der Befragung zeigen, dass planerisches Handeln in der regionalen Planungspraxis hauptsächlich durch formelle Aufgaben und Tätigkeiten bestimmt wird. Die Ergebnisse zeigen jedoch auch, dass informelle
Tätigkeiten ebenfalls einen wichtigen Bestandteil im Alltag eines Planers darstellen - und nach Einschätzung der Befragten zudem noch weiter an Bedeutung gewinnen werden (vgl. Kapitel 3.1). Auffallend ist, dass die Netzwerkarbeit, vor allem über die eigenen Grenzen hinweg, weder aktuell noch in der Zukunft als besonders wichtig eingestuft wird. Dabei geht es sowohl um überregionale Kooperationen als auch um internationale Kooperationen innerhalb der Planungspraxis. Anders verhält es sich mit Kooperationen zur Wissenschaft. Etwa $71 \%$ der Befragten geben diesbezüglich an, die Beteiligung an Forschungsprojekten als Quelle für Informationen zu nutzen bzw. genutzt zu haben (vgl. Kapitel 3.3). Was dabei jedoch auffällt, ist die Einstellung gegenüber dem wissenschaftlichen Diskurs. Demnach mangelt es am Transfer der wissenschaftlichen Erkenntnisse sowie an ihrer Praxisrelevanz. Die Mehrheit der Befragten ist sich zudem einig, dass die Erkenntnisse der Wissenschaft nicht oder nur zum Teil Antworten auf die Herausforderungen ihres Planungsalltages liefern und somit ,oft an der Realität vorbeigehen“. Zudem zeigen die Angaben über die Möglichkeiten und die Nutzung von Zugängen zur Wissenschaft, dass dieser nur eingeschränkt erfolgt. Obwohl eine Mehrheit der Befragten aktuell oder in der Vergangenheit an Forschungsprojekten beteiligt war bzw. ist, sind Forschungsberichte nur eine wenig genutzte Ressource, um Erkenntnisse aus der Wissenschaft zu erhalten. Es wird ein Missverhältnis zwischen in der praktischen Arbeit relevanten und wissenschaftlich besetzten Themen gesehen.

Wissenschaft und Praxis müssen in den direkten oder indirekten Austausch kommen, um bestmöglich voneinander profitieren zu können. Persönlicher Kontakt wird höher geschätzt als Bücher oder schriftliche Berichte. Dazu bedarf es Ressourcen wie Zeit, aber auch eine entsprechende finanzielle Ausstattung. Eine weitere Voraussetzung für einen gegenseitigen Profit von Planungswissenschaft und Planungspraxis sind das Verständnis und die Akzeptanz der Logiken und Erfolgskriterien des jeweils anderen Handlungsfeldes. Demnach müssen sowohl die Planungswissenschaft als auch die Planungspraxis die Ziele und Interessen des jeweils anderen verstehen und akzeptieren, dass eine Zusammenarbeit nicht immer profitabel ist oder sein muss. Das erfasste Meinungsbild der Regionalplaner hat gezeigt, dass die Nutzung und der Zugang zu wissenschaftlichen Erkenntnissen sowie eine Zusammenarbeit aus Sicht der Praktiker sinnvoll und erfolgreich eingeschätzt werden. Es gibt ein erkennbar gemeinsames Anliegen und gemeinsame Bezugspunkte, von denen eine strikte Trennung der drei Felder eher ablenkt (ähnlich Porter 2015: 295; vgl. Abbildung 1).

Whittemore (2015: 82) stellt aus einer umfangreichen Analyse US-amerikanischer Theoriebildung aus Wissenschaft und Praxis fest, dass in der Praxis eine sehr vielfältige Theorielandschaft vorherrscht, dass es nicht eine 
dominante Theorierichtung gibt und dass sich bestehende Theorien und neue Theoriebildung zur gleichen Zeit nicht ausschließen. Planungstheorien werden von den befragten Praktikern in ihrer Bedeutung wahrgenommen und befürwortet - sowohl in Bezug auf eine unmittelbare Unterstützung und Begründung des eigenen Handelns wie in der Reflexion und der kritischen oder kreativen Weiterentwicklung der Praxis. Die Planungstheorie scheint dennoch zu einseitig zu sein und möglicherweise zugunsten einer stärkeren Eindeutigkeit das Nebeneinander sehr unterschiedlicher Praktiken nicht ausreichend aufzunehmen (vgl. Whittemore 2015). Bei vielen scheint die Vorstellung vorzuherrschen, dass theoretische Ideen der Konzepte direkt in planerische Praxis übersetzt werden, was schon Alexander (1997: 5) kritisiert hat. Unklar ist hier aber, wie intensiv sich die Befragten mit Planungstheorien beschäftigen und welche Wege sie dazu nutzen. Die unterschiedlich intensive Nutzung von wissenschaftlichen Informationsquellen und vor allem die Schwierigkeiten mit längeren Berichten und Büchern (vgl. auch Taylor/Hurley 2016: 122 f.) können auch in Richtung eines Kommunikationsproblems interpretiert werden, wenn gerade neuere oder weniger prominente Ansätze in der Planungstheorie in der Praxis gar nicht erst wahrgenommen werden. Dennoch wird die Rolle von Planungstheorien als Kritik der Planungspraxis durch die Entwicklung von Gegenbildern oder durch die Erweiterung bestehenden Wissens mehrheitlich anerkannt. Praktiker scheinen durchaus gewillt zu sein, mit einer Vielfalt zu leben, sodass die Verfügbarkeit begründeter alternativer Theorien nicht negativ beurteilt werden muss, sondern vielmehr die Auswahl geeigneter Ansätze erst möglich macht (vgl. auch Allmendinger 2009: 24). Planungstheorie darf dann mindestens so vielfältig sein wie die inhärent interdisziplinäre Planungspraxis (vgl. Alexander 1997; Peters 2004: 5; Fainstein/DeFilippis 2016: $12 \mathrm{ff}$.). Mehr theoretische Vielfalt ist auch erforderlich, um die breit ausdifferenzierte Landschaft von Praktiken abzubilden. Eine bessere Verständlichkeit ist wichtig, um gemeinsam offene Stellen zu finden und die Entwicklung von Theorie und Praxis voranzubringen. Die hohe Bedeutung praktischer Restriktionen bedeutet aber auch, dass Planungstheorie und Planungspraxis nie identisch sein können, sondern die kreativen und imaginären Ideen, die zur Reflexion dienen können, immer ein Stück weit von praktischen Barrieren abstrahiert gedacht werden müssen.

\section{Was bleibt: Fragen und Herausforderungen für Wissenschaft und Theorie}

Dieser Beitrag soll und kann nicht die Frage danach beantworten, ob Planung ein definierter Tätigkeitsbereich ist, welche Aufgaben Theorien darin haben oder wie Planungs- praxis und Planungstheorie sich individuell oder gemeinsam weiterentwickeln können. Vielleicht ist Planung gleichzeitig alles oder nichts (vgl. Wildavsky 1973), vielleicht ist Planung auch ein theoretischer Sammelbegriff für sehr vielfältige und ortsspezifische Planungspraktiken (vgl. Vogelij 2015; Alexander 2016) und vielleicht gar ein gemeinsamer Glauben (Gunder/Hillier 2009: 8; vgl. auch Wildavsky 1973: 151 ff.). Diese Fragen können auf der Basis des hier präsentierten empirischen Materials an anderer Stelle aufgegriffen, weiterentwickelt und beantwortet werden. In diesem Beitrag erfolgt aus einer definierten Praxis heraus der Blick auf Wissenschaft und Theorie, um die Argumentation zu unterfüttern, die sich empirisch sonst eher auf Einzelfallstudien oder Absolventenstudien beziehen muss.

Es kann jedoch ohne Umschweife konstatiert werden, dass die Planungspraxis schon bei der Beschränkung auf den vergleichsweise engen Bereich der Regionalplanung vielfältiger ist, als es mit nur einer Theorie oder einer Gruppe von Theorien (z. B. kommunikativen Planungstheorien) beschrieben werden kann. Die Befragung der Praktiker in öffentlichen Regionalplanungsstellen in Deutschland hat gezeigt, dass auf Seiten der Praxis ein großes Interesse an wissenschaftlichen Erkenntnissen besteht und es bereits heute in Teilen einen intensiven Austausch mit der Wissenschaft gibt. Problematisch ist nach wie vor, dass die ,üblichen ' Produkte, mit denen die Wissenschaft in der Regel ihre Ergebnisse auf- und verbreitet (Bücher, Zeitschriftenaufsätze, Berichte von Forschungsprojekten etc.) in der Praxis nicht als erste und wichtigste Informationsquellen genutzt werden. In den Befragungsergebnissen wird sehr deutlich, dass die in der Praxis tätigen Personen wissenschaftliche Arbeiten und Erkenntnisse häufig aufgrund fehlender Praxisrelevanz als nicht nützlich einstufen. Das erfasste Meinungsbild hat zudem gezeigt, dass die Nutzung und der Zugang zu wissenschaftlichen Erkenntnissen ebenso wie eine aktive Zusammenarbeit aus Sicht der Praktiker sinnvoll und erfolgversprechend eingeschätzt werden.

Anhand der offenen Antwortmöglichkeiten innerhalb der Befragung wird deutlich, dass die Handlungsfelder Wissenschaft und Theorie von der Praxis oft wenig trennscharf voneinander abgegrenzt werden. Dies geschieht überraschenderweise selbst vor dem Hintergrund, dass auf Seiten der Praktiker ein teilweise umfassendes und eigenes Verständnis darüber besteht, was Theorien sind und was sie leisten sollen bzw. können. Weder Wissenschaftler noch durch sie formulierte Theorien sind in der Lage, die Bandbreite der Planungspraktiken abzudecken. Neben mehr Verständlichkeit - denn häufig scheitert ein effektiver Austausch zwischen Wissenschaft und Praxis an einfachen Kommunikations- oder Übersetzungsfehlern - ist demnach eine hohe Vielfalt erforderlich. Eine mögliche Orientierung können in diesem Zusammenhang vor allem gemeinsame Formate und Plattformen zum Austausch bieten. 
Für die weitere wissenschaftliche Auseinandersetzung wäre eine Wiederholung der Befragung für andere Planungsebenen und ein erweitertes Feld von Planern spannend. Eine breiter angelegte Befragung könnte erstens der Frage nachgehen, wie unterschiedlich Planungspraxis abhängig von der Planungsebene ausfällt. Und zweitens könnte nachvollzogen werden, ob es erkennbare Unterschiede nach Planungsaufgaben gibt, beispielsweise zwischen Wirtschaftsförderung, Stadt- und Regionalentwicklung und formeller Planung in Bauleitplanung und Regionalplanung. Damit könnten Schlussfolgerungen über die Sichtweisen der Praxis auf Wissenschaft und Theorie vergleichend erfasst und Empfehlungen zielgruppenspezifisch entwickelt werden.

\section{Literatur}

Alexander, E. R. (1997): A mile or a millimeter? Measuring the 'planning theory-practice gap'. Commentary. In: Environment and Planning B 24, 1, 3-6.

Alexander, E. R. (2010): Introduction: Does planning theory affect practice, and if so, how? In: Planning Theory 9, 2, 99-107.

Alexander, E. R. (2016): There is no planning - only planning practices. Notes for spatial planning theories. Essay. In: Planning Theory $15,1,91-103$.

Allmendinger, P. (2009): Planning theory. Basingstoke.

ARL - Akademie für Raumforschung und Landesplanung (2014): Raumentwicklung 3.0 - Thesen zur Zukunft der räumlichen Planung. Hannover. $=$ Positionspapier aus der ARL 95.

Beauregard, R. A. (2013): What Theorists Do. In: Urban Geography $33,4,474-487$.

Blotevogel, H. H.; Wiegand, T. S. (2015): Zur Evaluation von Wissensgenerierung und Wissenstransfer in der Akademie für Raumforschung und Landesplanung (ARL) - Leibniz-Forum für Raumwissenschaften. In: Raumforschung und Raumordnung 73, 3, 155-165.

Davoudi, S. (2015): Research impact. Should the sky be the limit? In Silva, E. A.; Healey, P.; Harris, N.; van den Broeck, P. (Hrsg.): The Routledge handbook of planning research methods. London, 405-413.

Fainstein, S. S.; DeFilippis, J. (2016): Introduction: The Structure and Debates of Planning Theory. In: Fainstein, S. S.; DeFilippis, J. (Hrsg.): Readings in planning theory. Chichester, 1-18.

Faludi, A. (1973): Planning theory. Oxford, New York. = Urban and Regional Planning Series 7.
Gunder, M.; Hillier, J. (2009): Planning in ten words or less. A Lacanian entanglement with spatial planning. Farnham.

Hurley, J.; Lamker, C. W.; Taylor, E. J.; Stead, D.; Hellmich, M.; Lange, L.; Rowe, H.; Beeck, S.; Phibbs, P.; Forsyth, A. (2016): Exchange between researchers and practitioners in urban planning: achievable objective or a bridge too far? Interface. In: Planning Theory and Practice 17, 3, 447-473.

Küsters, I. (2009): Narrative Interviews. Grundlagen und Anwendungen. Wiesbaden.

Lamker, C. W. (2016): Unsicherheit und Komplexität in Planungsprozessen. Planungstheoretische Perspektiven auf Regionalplanung und Klimaanpassung. Lemgo. = Planungswissenschaftliche Studien zu Raumordnung und Regionalentwicklung 6.

Lord, A. (2014): Towards a non-theoretical understanding of planning. In: Planning Theory 13, 1, 26-43.

MacDonald, K.; Sanyal, B.; Silver, M.; Ng, M. K.; Head, P.; Williams, K.; Watson, V.; Campbell, H. (2014): Challenging theory: Changing practice: Critical perspectives on the past and potential of professional planning. In: Planning Theory and Practice 15, 1, 95122.

Needham, B. (2004): John Friend: Advising and Theorizing. In: Planning Theory $3,3,237-247$.

Peters, D. (2004): Zum Stand der deutschsprachigen Planungstheorie. In Altrock, U.; Güntner, S.; Huning, S.; Peters, D. (Hrsg.): Perspektiven der Planungstheorie. Berlin, 5-18. = Reihe Planungsrundschau 10.

Porter, L. (2015): Unsettling comforting deceits. Planning scholarship, planning practice and the politics of research impact. Editorial. In: Planning Theory and Practice 16, 3, 293-296.

Renn, O. (2008): Anforderungen an eine integrative und transdisziplinäre Umweltforschung. In: Bergmann, M.; Schramm, E. (Hrsg.): Transdisziplinäre Forschung - Integrative Forschungsprozesse verstehen und bewerten. Frankfurt am Main, New York, 119-148.

Taylor, E. J.; Hurley, J. (2016): "Not a Lot of People Read the Stuff": Australian Urban Research in Planning Practice. In: Urban Policy and Research 34, 2, 116-131.

van de Ven, A. H.; Johnson, P. E. (2006): Knowledge for Theory and Practice. In: Academy of Management Review 31, 4, 802-821.

Vogelij, J. (2015): Is planning theory really open for planning practice? Comment. In: Planning Theory and Practice 16, 1, 128-132.

Whittemore, A. H. (2015): Practitioners Theorize, Too: Reaffirming Planning Theory in a Survey of Practitioners' Theories. In: Journal of Planning Education and Research 35, 1, 76-85.

Wildavsky, A. B. (1973): If Planning is Everything, Maybe it's Nothing. In: Policy Sciences 4, 2, 127-153.

Yiftachel, O. (1989): Towards a new typology of urban planning theories. In: Environment and Planning B 16, 1, 23-39.

Zierhofer, W.; Burger, P. (2007): Transdisziplinäre Forschung - ein eigenständiger Modus der Wissensproduktion? Problemorientierung, Wissensintegration und Partizipation in transdisziplinären Forschungsprojekten. In: Gaia 16, 1, 29-34. 